\title{
A Prototype of a Recommended Nutrition and Energy Expenditure Application for Smartphones
}

\author{
Kasikrit Damkliang, Member, IACSIT, Thakerng Wongsirichot, Jiraphon Saelue, and Juntamus Jingjit
}

\begin{abstract}
This paper proposes design and a prototype for a nutrition recommendation and energy expenditure application. The application runs on the iOS smartphones. The application advises users about daily calories intake and burn calculation and recommends the users how to exercise in order to balance the calories intake and burn. The application gives healthy calories intake and suitable exercise information and supports both of western and Thai cuisines. The application provides body mass index (BMI), basal metabolic rate (BMR), total energy expenditure (TDEE) and energy expenditure for exercise (EEE) estimation to the users that depends on their races and genders. The graphical user interface (GUI) design, full implementation, building, testing, and evaluation are also proposed. The application has achieved requirements and specifications. Finally, we have uploaded it into the App Store and freely distributing to the users with its name is "Calories iCare".
\end{abstract}

Index Terms-Nutrition recommendation, energy expenditure, physical activity, exercise, Thai cuisines, BMI, BMR, TDEE, smartphone.

\section{INTRODUCTION}

Nowadays, obesity is a major national issue in many countries, including Thailand as most of people are not aware of their nutrition and exercise. People tend to have excessive food energy intake whereas they may also have nutrition deficiency.

The lack of healthy nutrition and suitable exercise are significantly risk factors to invoke the obesity. It also links to related diseases such as the diabetes or the cardiovascular diseases. It may increase the risks to occur some cancers. In the contrast, the malnutrition influences growth and intelligent development in children. As a result, realizing in way of living and exercise is a significant thing in our life [1], [2], [3].

In previous works, there were many related health system such as a website that provided accurate information about diet and diabetes management in an interactive format, or the diabetes self-management learning system [4]. In addition, there is a large caring system integrating many components

Manuscript received September 1, 2014; revised February 15, 2015. This work was supported in part by a grant from Faculty of Science and Prince of Songkla University.

Kasikrit Damkliang and Thakerng Wongsirichot are with the Information and Communication Technology Programme, Faculty of Science, Prince of Songkla University, Hat Yai, Songkhla, Thailand (e-mail: \{kasikrit.d, thakerng.w\}@psu.ac.th)

Jiraphon Saelue and Juntamus Jingjit was with the Information and Communication Technology Programme, Faculty of Science, Prince of Songkla University, Hat Yai, Songkhla, Thailand (e-mail: \{5210210371, 5210210369\}@email.psu.ac.th). including mobile devices such as a mobile phones interfacing with the server [5]. At the present, smartphones are part of our daily life. Most people take their phones with them everywhere they go and utilize them to do many tasks more than any other communication devices. There are so many health application instances for smartphones such as a calorie counting and step walked application [6], [7]. These products are distributed for free and commercially via online places and market in both Apple and Android platforms. The most of them provide particularly in term of western cuisines. There has not yet been an application suggesting users using Thai cuisines. Although there is a website provided by the Institute of Nutrition, Mahidol University, it does not support mobile devices [8].

Our main objective of the application is to keep a healthy weight and living. This paper proposes the design and a prototype for a nutrition recommendation and exercise application. The application runs on the iOS smartphones. The application provides daily calories intake and burn calculation and recommends the users how to exercise in order to balance the calories intake and burn. In addition, the application gives healthy calories intake and suitable exercise information and supports both of western and Thai cuisines.

\section{RELATED ISSUES}

\section{A. Nutrition and Calories}

Nutritional status depends on nutrient quantity, which digested and aborted. There are differences between each person. Institute of Nutrition, Mahidol University suggests the nine qualitative guidelines in its Thai food based dietary guidelines (FBDGs).

Energy and nutrient consumption of the body for each day are changeable that depends on many conditions. In addition, nutrition also assumes to suitable foods for some kind of people, for example, athletes, pregnancy women or illness patients. A calorie is a unit of measure for energy and a pound is a unit of measure for mass, or weight. So then 1 pound is equal to 3,500 calories. Therefore, Total Daily Energy Expenditure (TDEE) is the total number of calories that our body expends in 24 hours, including all activities also known as maintenance level [9].

\section{B. Maintaining, Losing, and Gaining Weight}

It depends on a matter of calories input and output. To maintain weight. The American College of Sports Medicine recommends calorie levels never drop below 1,200 per day for women or 1,800 per day for men. For either gaining or losing weight, increasing or reducing calories by 15 to 20 percent above or below TDEE respectively, is a good 
suggestion [9]. The application provides the energy expenditure activity as well as the body exercise to users. The users can calculate and manage their optimal calories intake and energy expenditure to keep a healthy weight.

\section{METHOD TO MEASURE ENERGY EXPENDITURE}

\section{A. $B M I$}

A dietary goal is to keep body's weight to normal Body Mass Index (BMI) defined as the individual's body mass divided by the square of their height. The BMI category is shown in Table I [10], [11].

The BMI is an important value for evaluating body fats as it is easy to calculate and it can apply to all ranges of age. In Asian standard, the usual BMI of an adult is in between 18.5 to $22.9 \mathrm{~kg} / \mathrm{m}^{2}$. It is less than 18.5 to imply underweight and, equal or more than 23.0 to imply obese. We can indicate risk of diseases using our BMI value. If someone is obesity, he may have the risk of invoking Hypertension, Diabetes, or Heart Disease. On another hand, a severely underweight one may be endangered as he is easily infected and the physical fitness is reducing. Therefore, we should be keeping in the healthy weight.

\begin{tabular}{lll} 
& \multicolumn{2}{l}{ TABLE I: THE BMI CATEGORY } \\
\hline \hline Europe BMI & Asia BMI & Category \\
\hline less than 18.5 & Less than 18.5 & Underweight \\
18.5 to 24.9 & 18.5 to 22.9 & Normal (healthy weight) \\
$25.0-29.9$ & $23.0-24.9$ & Obesity level 1 \\
$30.0-34.9$ & $25.0-29.9$ & Obesity level 2 \\
$35.0-39.9$ & Equal or over 30 & Obesity level 3 \\
\hline \hline
\end{tabular}

\section{B. BMI and TDEE}

In our work, we also use Basal Metabolic Rate (BMR). The BMR is measured when human beings or other animals are at rest. It can be calculated by using Harris and Benedict formula [12] for men and women as shown in (1) and (2) respectively.

$$
\begin{aligned}
& 66.5+(13.75 * \mathrm{~kg})+(5.003 * \mathrm{~cm})-(6.775 * \text { age }) \\
& 655.1+(9.563 * \mathrm{~kg})+(1.850 * \mathrm{~cm})-(4.775 * \text { age }) .
\end{aligned}
$$

However, the BMR is an inconstant value affected by many factors including nutritional condition, pregnancy, surgery, infectious, athlete condition, level of gland hormones, genetics environment, etc.

In order to maintain current weight, it is simply need to manipulate physical activity levels and Total Energy Expenditure (TDEE). For example to determine BMR that a user is female with 30 years old, tall $167.6 \mathrm{~cm}$, weight 54.5 kilos. Then the BMR is $655+523+302-141$; it is 1339 calories per day. Next, we can determine a TDEE value; we suppose the user is moderately active (the factor level is 1.55). And then, the TDEE value is $1.55 * 1,339$; it is 2,075 calories per day [13].

\section{MET}

A Metabolic Equivalent (MET) reflects the energy expended by the body at rest. For example, running at $10 \mathrm{mph}$ (6 $\mathrm{min} / \mathrm{mile}$ pace) is equal to $14.5 \mathrm{METs}$ (i.e. 14.5 times more energy is required to run that pace than at rest). In related papers [6], [7], they detected daily activity only deskwork and walking MET values, not including physical activity or exercise. In this work, we deploy METs of physical and home activities for energy expenditure estimation from reference [14]. In case of all matters, the application will suggest exercise type and time spending. The energy expenditure is estimated using formula (3).

$$
0.0175 * \text { MET } * \text { weight } * \text { minute }=\mathrm{kcal}
$$

The 0.0175 value is a value of 1 MET, derived from 3.5 millimeters of oxygen per $\mathrm{kg}$ body weight per minute $(3.5 / 200)$. For example, a $55 \mathrm{~kg}$ person has 45 minutes aerobic (the MET of aerobic is 6.5), an energy expenditure value is $0.0175 \times 6.5 \times 55 \times 45$, and then it is $280 \mathrm{kcal}$.

\section{APPLICATION AND ARCHITECTURE AND DESIGN}

\section{A. Application Architecture}

The application is for running on the Apple's iOS devices, iPhone/iPad. We design the application in order to follow the principle of MVC and layer-based architecture considering the application functionality as shown in Fig. 1.

The application architecture divides into three components. It consists of GUI, Root View Controller (RVC) and, Data Source and Storage Engine (DSSE). The most important component is the RVC, which is responsible for application logical controlling and orchestrating all components to drive for the application goal.

The RVC orchestrates each UIView Controller which responses for each user interface object. The Energy Calculation Logic (ECL) module is in charge for logical core function of the application whereas life cycle and notification of the application are controlled by the Application Life Cycle Controller module (ALCC). In addition, the application provides sharing data to social network using the Facebook API.

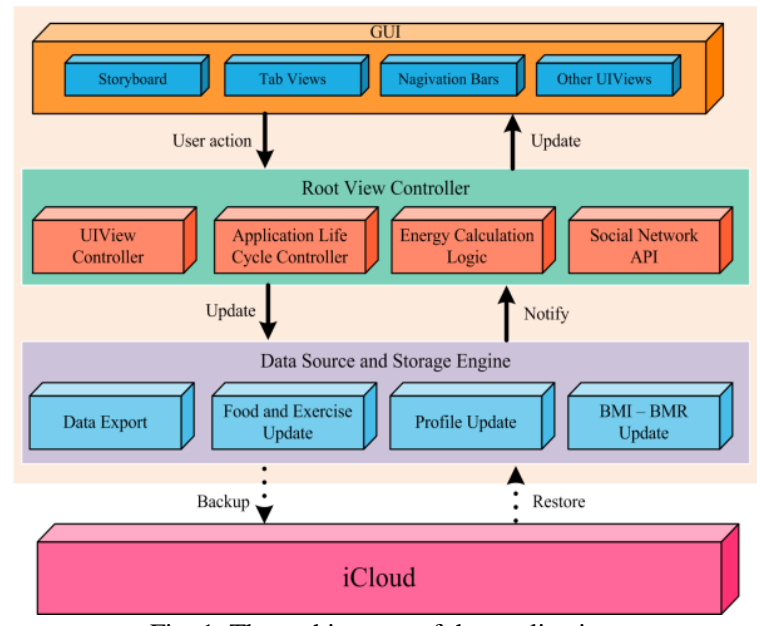

Fig. 1. The architecture of the application.

In case that user touches screen on an interface object, such as a navigation bar, the object sends the action to the UIView Controller. The controller then sends the update back to user interface such as changing page. On the other hand, if the user's action is an influence against data such as the user 
updates profile, the DSSE will then be in charge. In usual scenarios, the triggers may not only come from user interaction such as the ECL has detected the over energy consumption. Then, it will send message to the view controller and then, the view controller updates the user interface objects.

The application proposes personal usages on their own mobile smartphones. Even though, they login to the application on other devices, they are able to retrieve their profiles from cloud storages. In this work, we customize the Apple's iCloud to store the users' profiles.

\section{B. States of the Application}

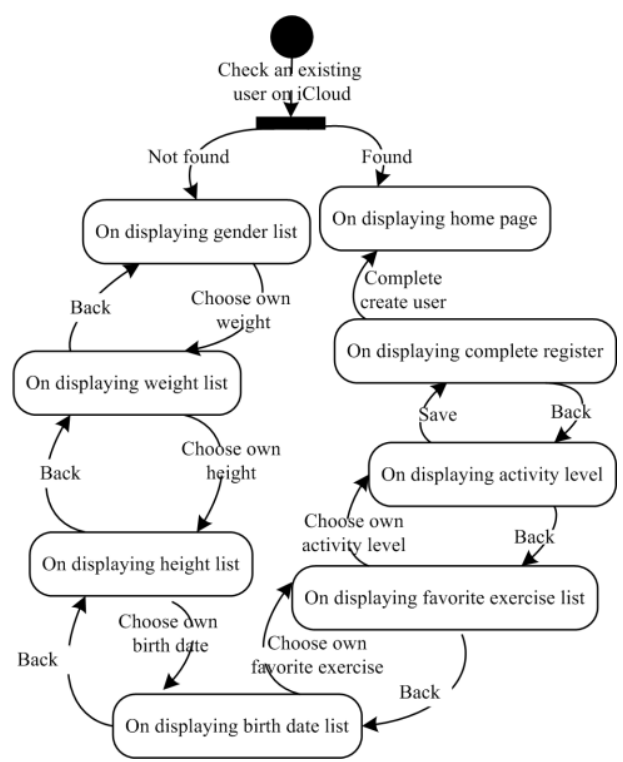

Fig. 2. The login and user's profile register state diagram.

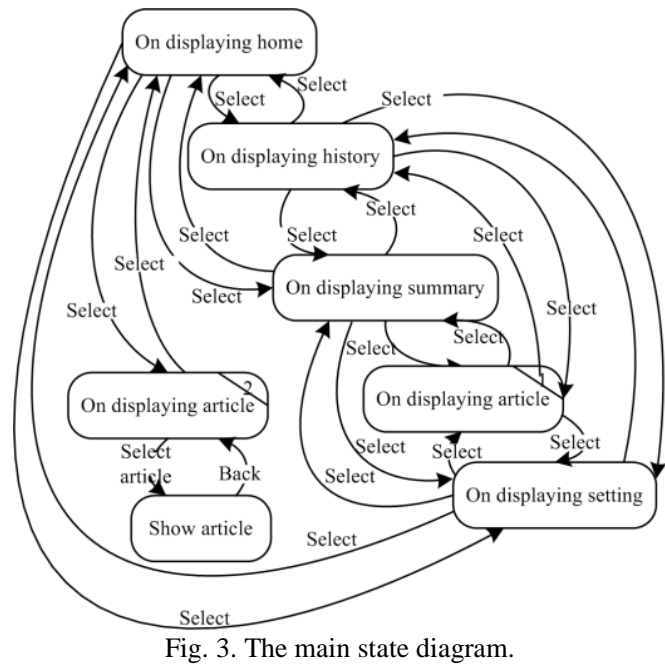

In our previous published paper [15], our application was a multi-view application. We have determined the number of views/pages and the interaction amongst them using state diagrams.

Login and user's profile register state is initialized as shown in Fig. 2. The application checks the user profile on iCloud. Then, if the application finds the existing profile, the application will restore the profile and display it on the home page. Otherwise, the application will process user registration asking for identification such as gender, height and birthdate. After that, it also leads the user to the home page. If a user has ended the application process from background, the application always invokes the main state for checking the user's profile. The iOS multitasking feature then resumes the last state of the application.

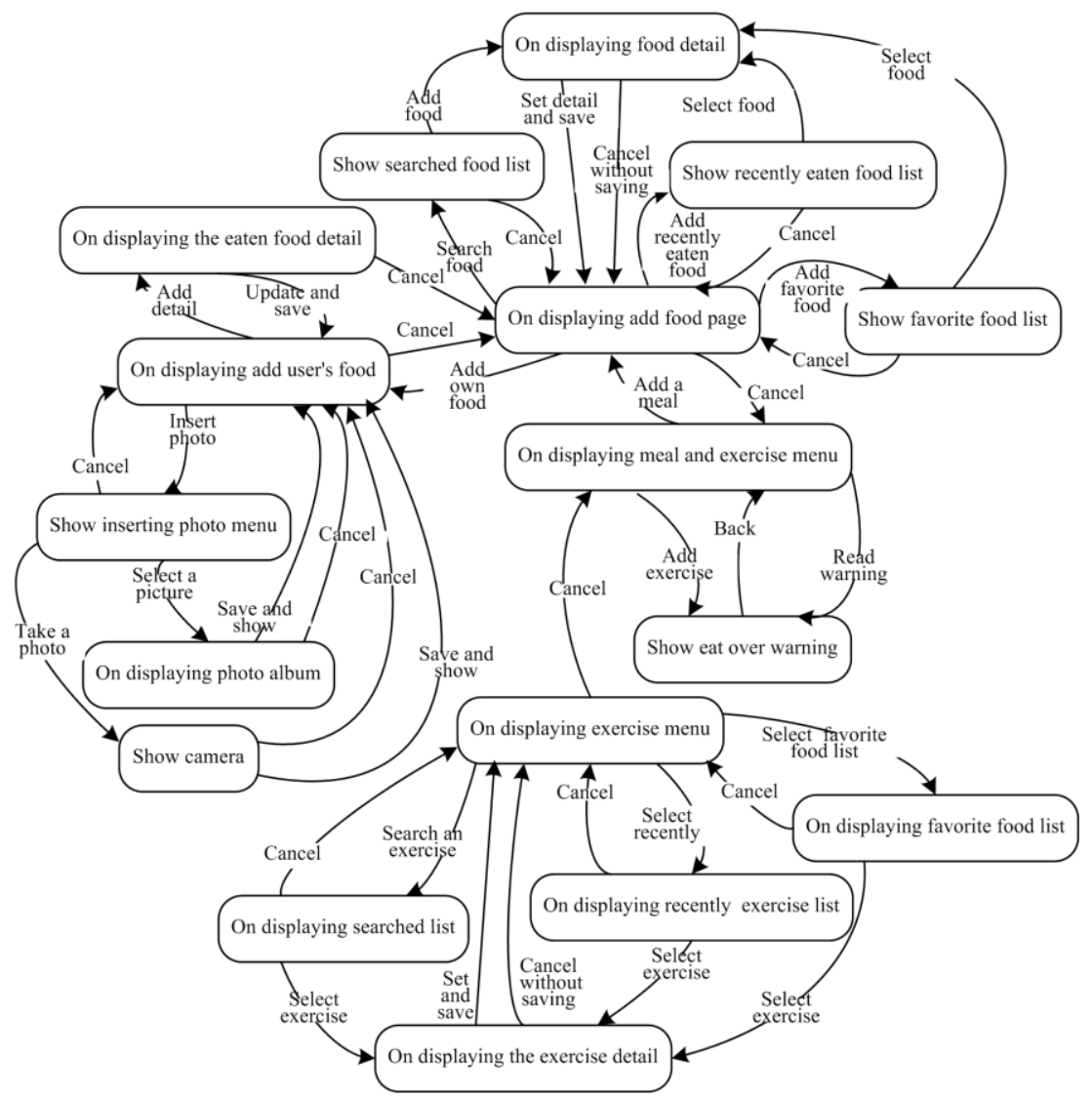

Fig. 4. The home page state diagram. 
Fig. 3 shows the overview state diagram of the application. The application consists of five taps including Home, History, Summary, Article and Setting. The Home page displays daily suitable food energy intake calculated from the user registered data. In addition, the user can insert foods/dishes and exercise that he actually takes. The History page provides the actual food energy intake and the exercise list. Finally, the Setting is for user configuration, for example, notification, profile setting, food list updating and, team developer contacts.

The functionality of the home page is shown in Fig. 4. A user can search and select some foods from the application list. The user can also select from his recent or favorite list. Furthermore, the application provides the user own define food and energy by themselves. The application supports the functionality list as well as the food list. The Exercise Recommendation will notify to the user when he has excessive daily food energy intake specified by the user selection in the registered profile.

The functionality of the home page is shown in Fig. 4. A user can search and select some foods from the application list. The user can also select from his recent or favorite list. Furthermore, the application provides the user own define food and energy by themselves. The application supports the functionality list as well as the food list. The Exercise Recommendation will notify to the user when he has excessive daily food energy intake specified by the user selection in the registered profile.

Fig. 5 shows the food and exercise history state diagram of the application. The application provides user eaten and exercise history list. The user can retrieve the details from a date on calendar. In addition, the user also can edit and update the lists. The application provides a user follow up weight and BMI in linear graph.

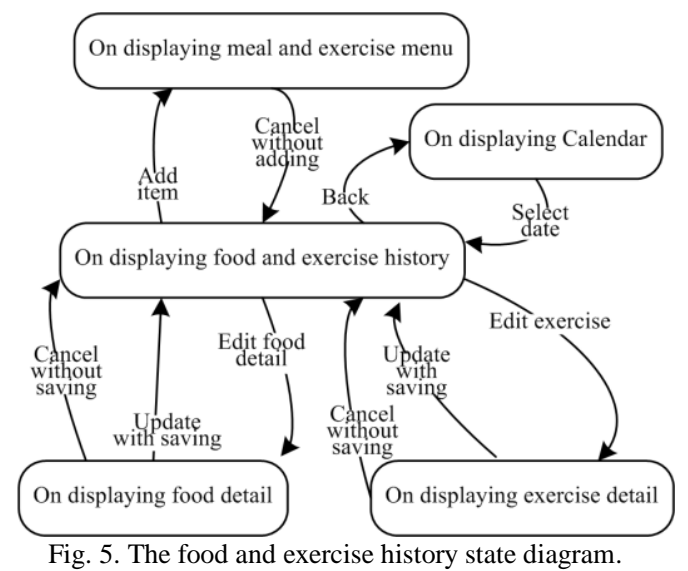

The application calculates the BMI with the changed weight from the user input. Moreover, the application displays energy in bar graph which compares the user consumed calories against to the previous 4 days energy that has been spent. The followed up weight and BMI state diagram is shows in Fig. 6.

The state diagram is for user configuration, the Setting page shown in Fig. 7. The first, notification is for alerting a user to record data and configure notify appearance style. The Next is for update the user's profile and, food and exercise list as the third. The application supports data backing up and exporting to external usage. Furthermore, the application provides

user's feedback mechanism. Finally, there are information about the application and $\log$ out function.

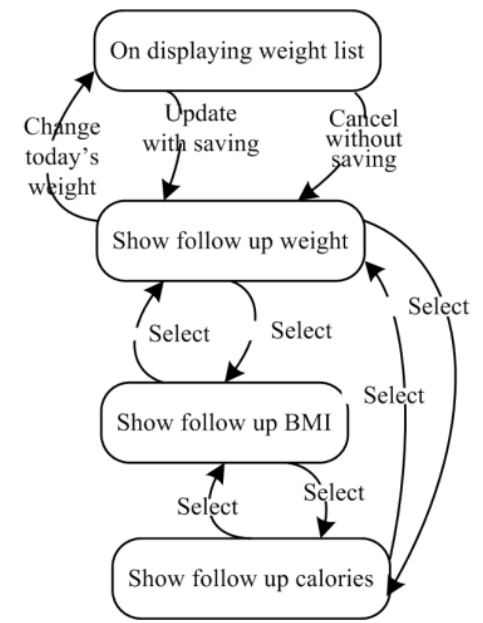

Fig. 6. The food and exercise history state diagram.

\section{BMI and TDEE Estimation}

In a point of view of the users, he will select his goal among maintaining, losing, or gaining weight. In this application, not only in the selected goal, the application will always recommend the user to proper exercise.

We propose Algorithm 1 for estimating all significant factors. When a user has started the application into the register process, the application calculates the user BMI to identify the level of body mass as shown in Algorithm 2 for BMI calculation. Then, the application will prompt to accept a weight in kilogram and a height in centimeter, age and gender to estimate the user's TDEE based from BMR as shown in Algorithm 3 for TDEE calculation.

The important data needed from the user in this application is the physical activity level in order to produce an activity multipliers which is various among $1.2,1.375,1.55,1.75$, and 1.90 and depends on the user's specific physical status. Hence, the user should give the suites activity level into the application. In addition, if the exercise frequency changes, the user can also re-adjust the activity level.

\footnotetext{
Algorithm 1 mainEstimation

Require: Algorithms calBMI, calTDEE, calEEE and calRemainEEE

Define: actualCI=Actual Calory Intake, optimalCI = Optimal Calories

Intake, optimalEEE = Optimal Energy Expenditure for Exercise, TDEE =

Total Daily Energy Expenditure

1. Start

2. Read actualCI, weight $(\mathrm{kg})$, height $(\mathrm{cm})$, age, gender

3. Read sport, time, remainEEE

4. Set bmiLevel $=$ calBMI(weight, height)

5. Set TDEE = calTDEE(weight, height, age, gender)

6. Set exerciseLevel $=$ calOptimalEEE(bmiLevel, TDEE)

7. Set remainEEE = calRemainEEE( sport, time, weight, optimalEEE)

8. Set optimalEEE $=$ remainEEE + optimalEEE

9. Display optimalCI, actualCI, actualEEE

10. Stop
}

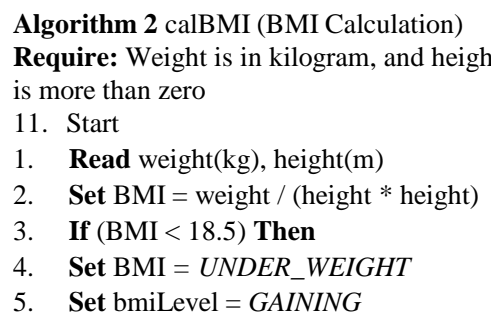


6. Else If $(\mathrm{BMI}>=18.5$ And $\mathrm{BMI}<=22.9)$ Then

7. Set $B M I=H E A L T H Y \_W E I G H T$

8. Set bmiLevel $=$ MAINTAIN

9. Else If $(\mathrm{BMI}>=23.0$ And $\mathrm{BMI}<=24.9)$ Then

10. Set $B M I=O V E R \_W E I G H T$

11. Set bmiLevel $=L O S I N G$

12. Else If $(B M I>=25.0$ And $B M I<=29.9)$ Then

13. Set $B M I=O B E S E$
14. Set bmiLevel $=$ LOSING

15. Else If $(B M I>30)$ Then

16. Set $B M I=S E V E R E L Y \_O B E S E$

17. Set bmiLevel $=$ LOSING

18. End If

19. Return bmiLevel

20. Stop

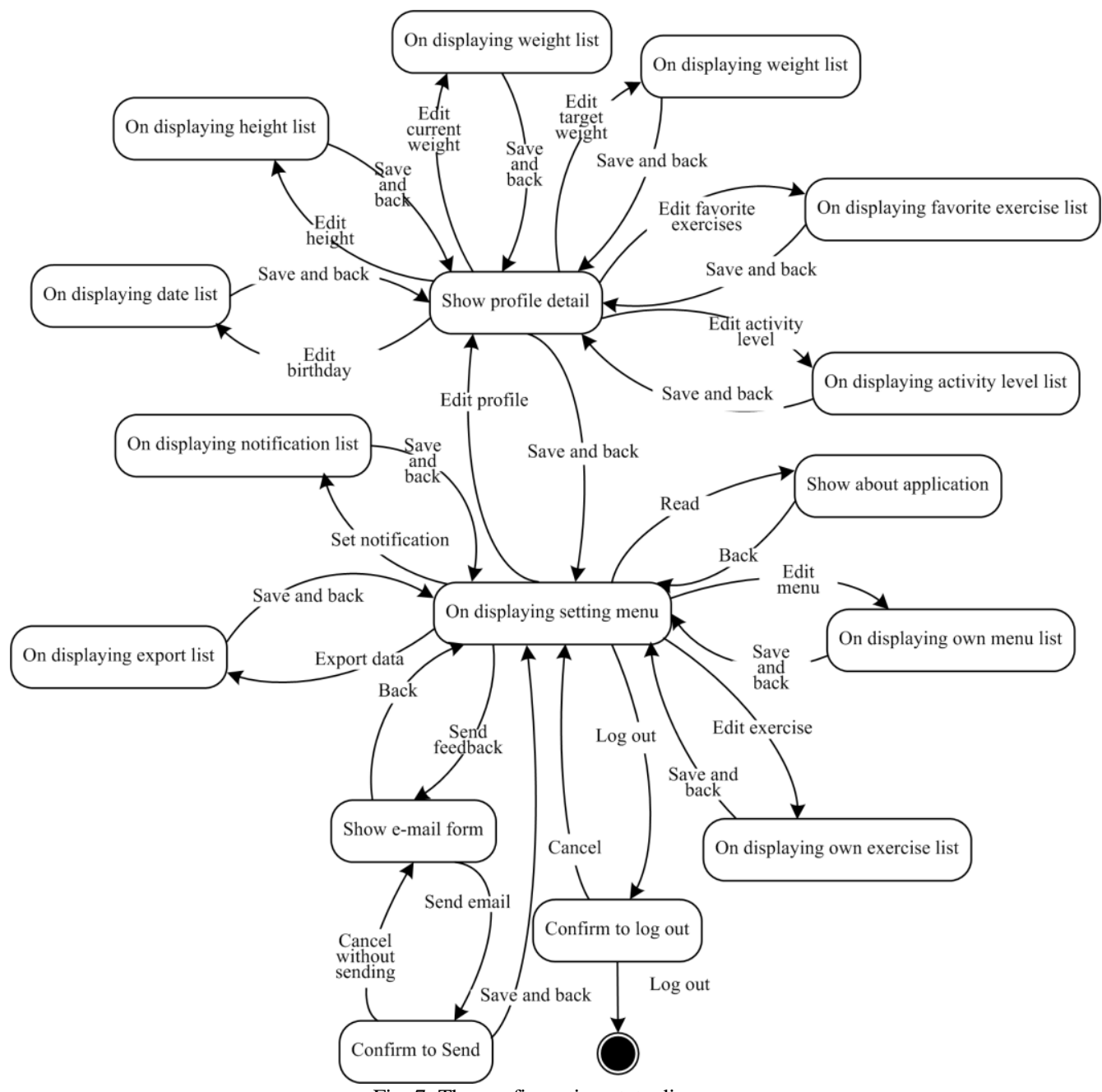

Fig. 7. The configuration state diagram.

Algorithm 3 calTDEE (Total Daily Energy Expenditure Calculation) Require: Weight is in kilogram and height is in meter in which each value is more than zero

Require: Age calculated by birthdate and gender is FEMALE or MALE.

1. Start

2. Read weight $(\mathrm{kg})$, height $(\mathrm{m})$, age, sex, activityLevel

3. If (gender $=$ FEMALE) Then

4. Set $B M R=655.1+9.563 *$ weight $+1.850 *$ height $-4.775 *$ age

5. Else

6. Set $B M R=66.5+13.75 *$ weight $+5.003 *$ height $-6.775 *$ age

7. End If

8. If (activityLevel $=S E D E N T A R Y)$ Then

9. Set $T D E E=B M R * 1.20$

10. Else If (activityLevel $\left.=L I G H T L Y \_A C T I V E\right)$ Then

11. Set $T D E E=B M R * 1.375$

12. Else If (activityLevel $=$ MODETRATELY_ACTIVE) Then

13. Set $T D E E=B M R * 1.55$

14. Else If (activityLevel $\left.=V E R Y \_A C T I V E\right)$ Then

15. Set $T D E E=B M R * 1.725$

16. Else If (activityLevel $\left.=E X T R A \_A C T I V E\right)$ Then

17. Set $T D E E=B M R * 1.90$

18. End If

19. Return TDEE

20. Stop

\section{Energy Expenditure for Exercise (EEE) Estimation}

We consider the calculation into three schemas for each goal between optimal calories intake (OCI) and actual calories intake (ACI). The first schema is maintaining weight, the user should have the OCI which equals to the TDEE and should exercise normally 3 to 5 days a week. In case of the $\mathrm{ACI}$ is less than or equal the OCI for at least 3 days, the application will recommend the user to increase calories intake and to exercise 1 to 2 days a week. The second schema is losing weight, the user should decrease the OCI to 15 percent of the TDEE and should exercise daily 6 to 7 days a week. In case of ACI is less than or equal the OCI, we configure base value of the EEE to 10 percent of TDEE for motivating the user to have slightly exercise. The last schema is gaining weight; the user should increase the OCI to 15 percent of the TDEE and exercise slightly 1 to 3 days a week.

Algorithm 4 shows proposed algorithms for calculating optimal food energy intake and energy expenditure for exercise (EEE). If the user has excessive calories intake, the application will recommend suitable exercises depending on 
the kind of sports and time spending which consults the

Algorithm 5 shows proposed algorithms for calculating actual EEE and remaining EEE from user's physical exercise with the METs. In case of losing weight, even though the user have burnt calories exceed the optimal EEE, but the application will determine the remaining EEE to zero because our body cannot burn calories above or below TDEE by 15 to 20 percent [9].

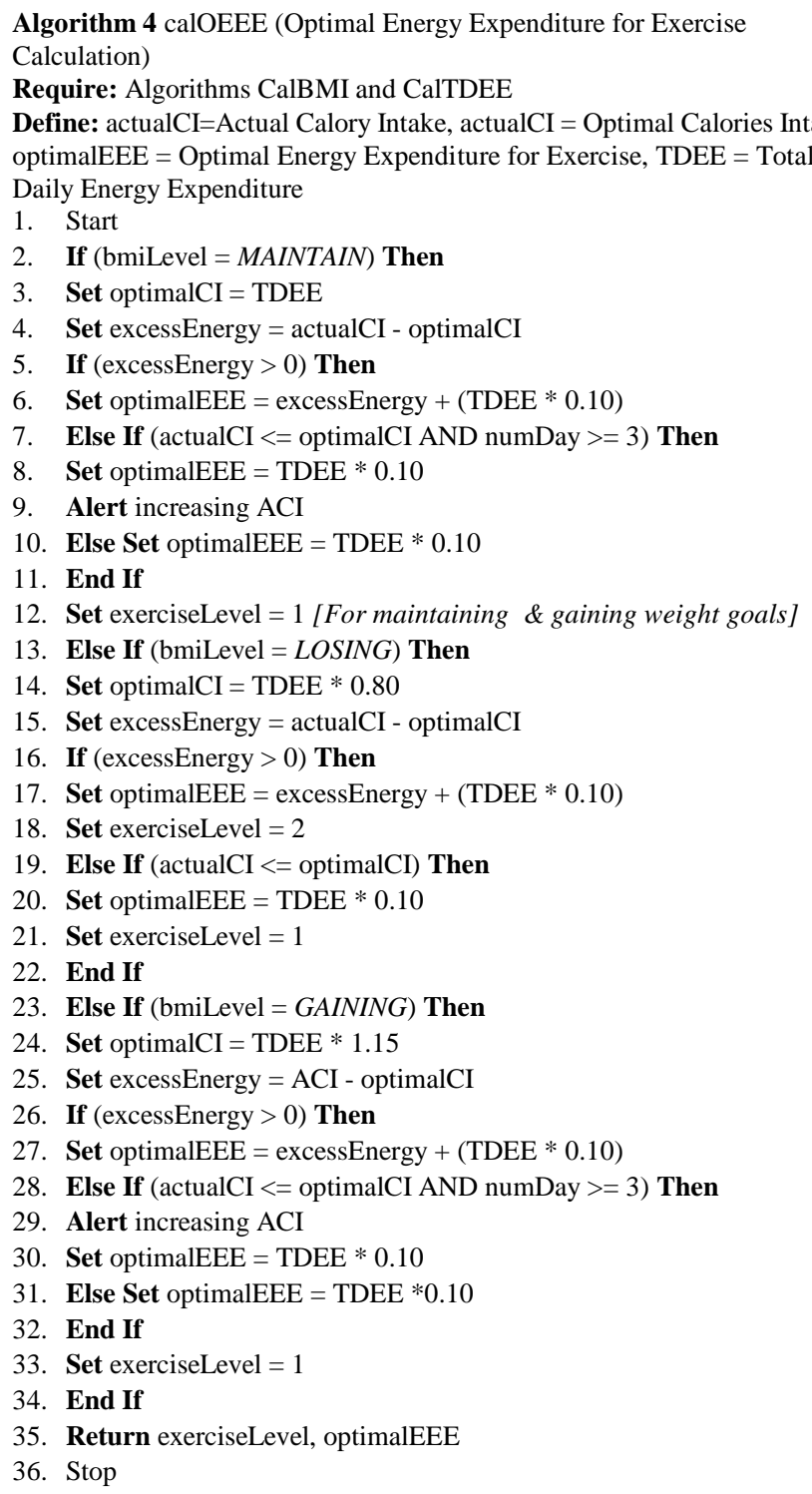

\section{Algorithm 5 calRemainEEE}

Define: actualCI=Actual Calory Intake, optomalCI $=$ Optimal Calories Intake, optimalEEE = Optimal Energy Expenditure for Exercise.

1. Start

2. Set actualEEE $=0.0175 *$ weight $*$ time $*$ MET

3. If $($ exerciseLevel $=1)$ Then

4. If (actualEEE $>=0$ AND actualEEE $<=$ optimalEEE) Then

5. Set remainEEE $=$ optimalEEE - actualEEE

6. Go to 18

7. Else

8. Set remainEEE $=0$

9. Go to 18

10. End If

11. Else

12. Set remainEEE $=$ optimalEEE - actualEEE

13. If (remainEEE < 0 ) Then

14. Set remainEEE $=0$

15. End If

16. Go to 18

\author{
17. End If \\ 18. Return remainEEE \\ 19. Stop
}

\section{E. Prototype Design of GUI}

The main page of the application also shows the daily OCI and optimal EEE with a graphical pie chart. The chart is presented with difference colors indicated amount of the both values. The GUI shows the user's calories intake during a day time, the chart will be dynamically changing that depends on the user's ACI and actual EEE as shown in Fig. 8(a) and (b) respectively.

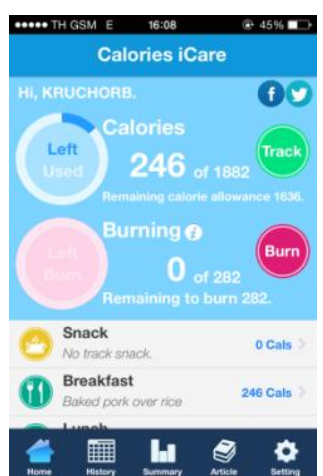

(a)

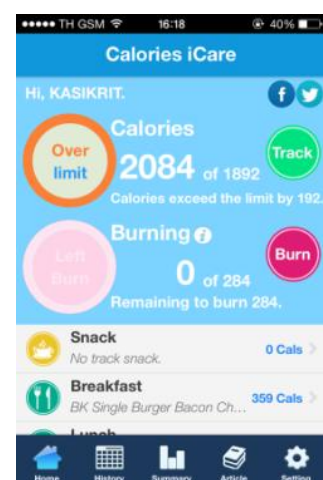

(b)
Fig. 8. The GUI Design of optimal and actual of calories intake and EEE.

In scenario of the user's EEE estimation, the application will recommend the fitting of the EEE by various kind of sport and its' calories burning. However, the user can adjust own exercise list by selecting provided sport list in the application. The user also can change time spending of each sport. Then, the application will re-estimate the EEE and summarize results to the user with the GUI as shown in Fig. 9(a) and (b) consequently

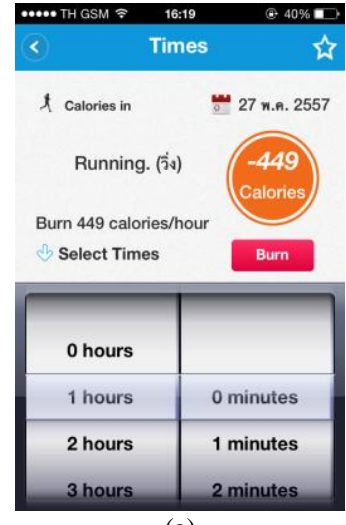

(a)

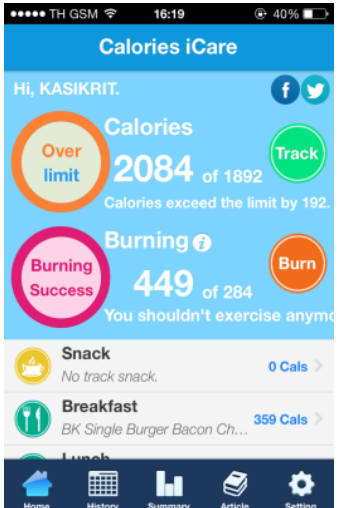

(b)
Fig. 9. The GUI design of re-estimate the EEE and summarize results.

\section{IMPLEMENTATION AND DISTRIBUTION PLATFORM}

\section{A. Implementation, Building and Testing}

We reveal the calories intake and burn information using the Storyboard which is provided by the UIKit. The powerful Storyboard is for the multiview-based navigation application. It supports table view and developers can customize the table cells. In this work, we implement the pages with Tab bar and link all pages together. The application utilizes the Core 
Services for storing pre-processed data such as the user profile, eating and exercise data as shown in Fig. 10. The application supports the iOS PLIST data structure for storing defined data. We have built and tested the application on the iPhone simulator and its real devices with the iOS 6.0. The application can calculate caries intake and energy expenditure correctly.

\section{B. Distribution Platform}

The application supports the iOS version 6.0 or later with a trademark Apple smartphone. The application also compatibly runs on iPad. We have uploaded it into the App Store and freely distributing to the users with its name is "Calories iCare".

\begin{tabular}{|c|c|c|}
\hline Profile & Eating & Exercise \\
\hline $\begin{array}{l}\text { T Attributes } \\
\text { activity } \\
\text { bdate } \\
\text { editdate } \\
\text { gender } \\
\text { height } \\
\text { username } \\
\text { weight }\end{array}$ & $\begin{array}{l}\text { T Attributes } \\
\text { datetime } \\
\text { f_calorie } \\
\text { f_meal } \\
\text { f_name } \\
\text { f_nameth } \\
\text { f_serving } \\
\text { f size }\end{array}$ & $\begin{array}{l}\text { TAttributes } \\
\text { datetime } \\
\text { ex_met } \\
\text { ex_name } \\
\text { ex_nameth } \\
\text { ex_time } \\
\text { ex_weight } \\
\text { Velationships }\end{array}$ \\
\hline Relationships & f_weight & \\
\hline
\end{tabular}

Fig. 10. The Core Service data dictionary of pre-processed data.

\section{FUTURE WORK}

The application can utilizes new features of the iOS 8. The application will make the system more personalized such as different schedules to loose fat, gain weights or build muscles. In addition, the user can add weekly planner to set up a goal

\section{CONCLUSION}

The main objective of the application is to keep the users' healthy weight. This paper proposes a prototype for a nutrition recommendation and energy expenditure application. The application advises the user about the food energy intake and suitable energy consumption on exercise in daily life. Thai dishes are suggested in this work. The application supports the iOS version 6.0 or later on the Apple smartphones. The GUI design, implementation, building, testing, and evaluation are also proposed. The application has achieved requirements and specifications.

\section{ACKNOWLEDGMENT}

The authors wish to thank Assistant Prof. Dr. Pichaya Tandayya for proof reading the paper. This work was supported in part by a grant from Faculty of Science and Prince of Songkla University.

\section{REFERENCES}

[1] N. Rojroongwasinkul, V. Kosulwat, T. Viriyapanich, A. Boonpraderm, and K. Wanijjakul, "Nutrient adequacy in Thai elderly," presented at the 5th Asian Congress of Dietetics, Bangkok, Thailand, November $10-12,2010$.
[2] M, Hassapidou and S. K. Papadopoulou, "Food choice criteria in adodescents according to body image," Songklanakarin J. Sci. Technol., vol. 28, pp. 165-169, 2006.

[3] W. Thasanasuwan, "Obesity in children is more in urban than in runal: Are really less active?" presented at the International Conference on Childhood Obesity, Oeiras, Portugal, July 6-9, 2011.

[4] K. Herrejon, J. L. Hartke, J. Scherer, and K. Chapman-Novakofski, "The creation and impact evaluation of your guide to diet and diabetes, an interactive web-based diabetes tutorial," The Journal of Diabetes Technology and Therapeutics, vol. 11, issue 3, pp. 17-179, Mar. 1, 2009.

[5] M. Glasemann and A. M. Kanstrup, "IT for learning diabetes," Stud. Health Technol. Inform, vol. 157, pp. 154-159, 2010.

[6] N. Ryu, Y. Kawahawa, and T. Asami, "A calories count application for a mobile phone based on METS value," in Proc. the Sensor, Mesh and Ad Hoc Communications and Networks (SECON 2008), June 16-20, 2010, pp. 583-584.

[7] A. Khalil and S. Glal, "Stepup: A step counter mobile application to promote healthy lifestyle," in Proc. the Current Trends in Information Technology, Dec. 15-16, 2009, pp. 1-5.

[8] U. Chantima, S. Taechangam, C. Pachotikarn, and W. Senavongse, "Development of a multimedia website educational tool for self-help meal planning using a carbohydrate counting concept in type 1 diabetic adolescents," presented at the 5th Asian Congress of Dietetics, Bangkok, Thailand, November 10-12, 2010.

[9] P. P. Sirichakwal, K. Sranacharoenpong, and K. Tontisirin, "Food based dietary guidelines (FBDGs) development and promotion in Thailand," Asia Pacific Journal of Clinical Nutrition, vol. 20, no. 3 , June 15, 2011

[10] Division of Physical Activity and Health, Department of Health, Ministry of Public Health. (August 2014). BMI: Body mass index. [Online]. Available: http://dopah.anamai. moph.go.th/bmi.php

[11] Bureau of Nutrition, Department of Health, Ministry of Public Health. (August 2014). [Online]. Available: http://nutrition.anamai.moph.go.th /temp/main/index.php

[12] J. Harris and F. Benedict, A Biometric Study of Basal Metabolism in Man, Carnegie Institute of Washington, Washington D.C., 1919.

[13] B. A. Bushman, "How can I use METS to quantify the amount of aerobic exercise," ACSM's Health \& Fitness Journal, vol. 16, no. 2, pp. 5-7, 2012.

[14] A. C. P. Volp, F. C. E. de Oliveira, R. D. M. Alves, E. A. Esteves, and J. Bressan, "Revision energy expenditure: Component and evaluation methods," The Journal of Nutr. Hosp., vol. 26, vol. 3, pp. 430-440.

[15] K. Damkliang and P. Anachotikul, "Application prototype of children medicine taking notification on mobile devices," in Proc the ECTI-CARD 2014, Chiangmai, Thailand, May 21-23, 2014, pp. D105-D110.

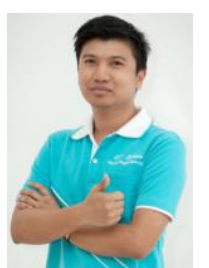

Kasikrit Damkliang received the B.S. degree in computer science in 2005 and M.Sc. degree in computer engineering in 2009 from the Prince of Songkla University, Thailand.

$\mathrm{He}$ is currently a lecturer in the Information and Communication Technology Programme, Faculty of Science and currently is also a PhD student at the Department of Computer Engineering, Faculty of Engineering, Prince of Songkla University, Thailand. His research interests includes mobile application developments, workflow and cloud computing, HPC, web services and bioinformatics.

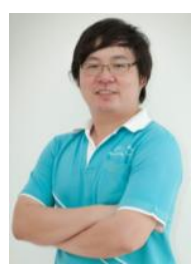

Thakerng Wongsirichot is an assistant professor specialized in information technology. He received the B.Com. degree in e-commerce and business information systems and the M.I.S. degree from the University of Wollongong, Australia.

$\mathrm{He}$ is currently a lecturer in the Information and Communication Technology Programme, Faculty of Science, Prince of Songkla University, Thailand His research interests includes mobile application developments, e-commerce, and medical information systems. 